Cahiers de recherches médiévales

\title{
« Noter biaus chans por ramenbrance de chançons 》)
}

Les auteurs narratifs font-ils œuvre de témoins de leur temps musical ?

\section{Anne lbos-Augé}

\section{(2) OpenEdition}

\section{Journals}

Édition électronique

URL : https://journals.openedition.org/crm/773

DOI : $10.4000 / \mathrm{crm} .773$

ISSN : 1955-2424

\section{Éditeur}

Honoré Champion

Édition imprimée

Date de publication : 30 décembre 2006

Pagination : 257-270

ISSN : 1272-9752

Référence électronique

Anne Ibos-Augé, « "Noter biaus chans por ramenbrance de chançons » », Cahiers de recherches médiévales [En ligne], 13 | 2006, mis en ligne le 27 novembre 2009, consulté le 15 décembre 2022. URL : http://journals.openedition.org/crm/773 ; DOI : https://doi.org/10.4000/crm.773 


\title{
rin
}

\section{« Noter biaus chans por ramenbrance de chançons »: les auteurs narratifs font-ils ouvre de témoins de leur temps musical?}

\author{
Cil qui mist cest conte en romans, \\ ou il a fet noter biaus chans \\ por ramenbrance des chançons, \\ veut que ses pris et ses renons \\ voist en Raincïen en Champaigne \\ et que li biaus Miles l'apregne \\ de Nantuel, uns des preus del regne; \\ car aussi com l'en met la graine \\ es dras por avoir los et pris, \\ einsi a il chans et sons mis \\ en cestui Romans de la Rose, \\ qui est une novele chose \\ et s'est des autres si divers \\ et brodez, par lieus, de biaus vers \\ que vilains nel porroit savoir. ${ }^{1}$
}

Par ces vers, Jean Renart commence le prologue de son roman de Guillaume de Dole. Outre la volonté de nouveauté avérée au douzième vers, la revendication historique est clairement établie dès les second et troisième vers : il s'agit pour l'écrivain de «noter» les chansons, c'est-à-dire d'en consigner la musique, afin qu'elles demeurent dans la mémoire des lecteurs de son roman. Le procédé, nouveau pour les œuvres narratives ${ }^{2}$, se répètera dans de nombreux textes tout au long du $\mathrm{XIII}^{\mathrm{e}}$ siècle et jusqu'au début du $\mathrm{XIV}^{\mathrm{e}}$, dépassant d'ailleurs largement le cadre du roman. Si Gautier de Coinci, pour les œuvres didactiques, Jean Renart et Gerbert de Montreuil, pour les romans, sont les seuls à mentionner dans leurs textes leur intention de les farcir de chansons, nombre d'écrivains ont en effet inclus des pièces lyriques dans leurs œuvres. Celles-ci peuvent être complètes ou fragmentaires et comporter ou non une notation musicale sur les témoins les conservant: quel que soit leur état dans le texte qui les cite, elles sont des témoins de l'histoire musicale. Et cette dernière possède de surcroît deux réalités temporelles : celle de la citation et celle, souvent plus tardive, de son œuvre-hôte. C'est cette double réalité historique que je me propose d'examiner ici, afin de tenter de cerner les limites de cette mémoire dont parlait Jean Renart. Je me baserai sur les chansons courtoises,

\footnotetext{
${ }^{1}$ Jean Renart, Le roman de la rose ou de Guillaume de Dole, éd. par Félix Lecoy, Paris, Champion, 1979, v. 1-15.

${ }^{2}$ En 1228 , date actuellement généralement admise pour le roman, des sermons latins ont déjà utilisé la lyrique comme niveau supplémentaire de discours: des rondeaux français sont parfois mis en exergue des sermons et réutilisés dans le cours du texte, à la manière d'exempla. On consultera avec profit à ce sujet l'ouvrage de Michel Zink, La prédication en langue romane avant 1300, Paris, Champion, 1982. Gautier de Coinci insère également quelques-unes de ses propres compositions dans ses Miracles, dès 1223-1227.
}

Cahiers de Recherches Médiévales (XII ${ }^{e}-X V^{e}$ s.), 13, 2006 
généralement plus aisées à dater que leurs homologues à danser, et dont, d'ailleurs, les auteurs sont parfois mentionnés dans les œuvres.

Si les poèmes insérés par Gautier de Coinci dans ses Miracles sont bien de lui, il a eu souvent recours, pour les mettre en musique, au phénomène du contrafactum, courant chez les trouvères et qui consiste à adapter un texte nouveau sur une mélodie préexistante. Outre les sources liturgiques, difficiles à rattacher à un temps précis, et les auto-plagiats, les emprunts aux trouvères sont nombreux parmi les chansons citées dans le recueil marial. En voici une liste récapitulative, assortie de quelques remarques formelles qui permettront parfois de dégager les parentés musicales réelles de certaines des chansons, et donc d'en identifier les auteurs originels :

\begin{tabular}{|c|c|c|c|c|c|}
\hline $\begin{array}{c}\mathrm{N}^{\circ} \text { d'ordre et } \\
\text { incipit }\end{array}$ & $\begin{array}{l}\text { Nbre de } \\
\text { groupes } \\
\quad \text { de } \\
\text { versions }\end{array}$ & Mode & Ambitus & Forme & $\begin{array}{c}\text { Remarques } \\
\text { musicales }\end{array}$ \\
\hline $\begin{array}{l}\text { 1-Amors qui seit } \\
\text { bien enchanter }\end{array}$ & 2 & $\begin{array}{l}\text { Fa authente à } \\
\text { hauteur d'ut } \\
\text { Sol plagal }\end{array}$ & $\begin{array}{l}8 \mathrm{j} \\
5 \mathrm{j}\end{array}$ & $\begin{array}{l}\text { ABCDEF } \\
\text { ABCB'B', A' }\end{array}$ & $\begin{array}{l}\text { Version en } f a \\
\text { authente } \\
\text { semblable à Sour } \\
\text { cest rivage }\end{array}$ \\
\hline $\begin{array}{l}\text { 2-Qui que face } \\
\text { rotruenge } \\
\text { novele }\end{array}$ & 3 & $\begin{array}{l}\text { Fa authente } \\
\text { Ré plagal } \\
\text { Ré authente } \\
\text { ou fa authente }\end{array}$ & $\begin{array}{l}8 \mathrm{j} \\
9 \mathrm{M} \\
9 \mathrm{M} \\
\text { étendue à } \\
10 \mathrm{M} \text { ou } \\
7 \mathrm{~m} \\
\text { étendue à } \\
8 \mathrm{j}\end{array}$ & $\begin{array}{l}\text { ABABCDEFF' } \\
\text { ABABCDEC'C', } \\
\text { ABCDEFGHI }\end{array}$ & $\begin{array}{l}\text { Version en ré } \\
\text { plagal: } \\
\text { contrafactum de } \\
\text { R } 482\end{array}$ \\
\hline $\begin{array}{l}\text { 6-Quant ces } \\
\text { floretes florir } \\
\text { voi }\end{array}$ & 1 & $\begin{array}{l}\text { Sol plagallSol } \\
\text { plagal } \\
\text { finissant en ré }\end{array}$ & $\begin{array}{l}7 \mathrm{M} \\
\text { étendue à } \\
8 \mathrm{j}\end{array}$ & AABCDD' & $\begin{array}{l}\text { Les versions } \\
\text { diffèrent } \\
\text { légèrement par } \\
\text { la fin } \\
\text { Contrafactum de } \\
\text { R } 2030\end{array}$ \\
\hline $\begin{array}{l}\text { 9-Sour cest } \\
\text { rivage, a ceste } \\
\text { crois }\end{array}$ & 1 & $\begin{array}{l}\text { Fa plagal à } \\
\text { hauteur d'ut }\end{array}$ & $8 \mathrm{j}$ & $\mathrm{ABCDEF}$ & $\begin{array}{l}\text { Même musique } \\
\text { que le 1er } \\
\text { groupe de } \\
\text { versions de } \\
\text { Amors qui seit } \\
\text { bien enchanter }\end{array}$ \\
\hline $\begin{array}{l}\text { 10-De sainte } \\
\text { Leocade }\end{array}$ & 1 & Sol authente & $\begin{array}{l}8 \mathrm{j} \text { étendue } \\
\text { à } 9 \mathrm{M} \text { ou } \\
10 \mathrm{~m}\end{array}$ & ABABCDEF & $\begin{array}{l}\text { Même musique } \\
\text { que } \mathrm{R} 83 \text {, } \\
\text { Entendez tuit } \\
\text { ensemble, aussi } \\
\text { de Gautier, mais } \\
\text { pas insérée dans } \\
\text { les Miracles }\end{array}$ \\
\hline
\end{tabular}




\begin{tabular}{|c|c|c|c|c|c|}
\hline $\begin{array}{l}\text { 11-Pour la } \\
\text { pucele en } \\
\text { chantant me } \\
\text { deport }\end{array}$ & 2 & $\begin{array}{l}\text { Ré plagal et } \\
\text { authente }\end{array}$ & \begin{tabular}{|l}
$6 \mathrm{M}$ \\
étendue à \\
$7 \mathrm{~m}$ \\
$8 \mathrm{j}$ étendue \\
à $9 \mathrm{M}$ \\
\end{tabular} & $\begin{array}{l}\text { AA'AA'BB'CC' } \\
\text { ababcdef }\end{array}$ & $\begin{array}{l}\text { Version en sol } \\
\text { authente: } \\
\text { contrafactum de } \\
\text { R } 1429\end{array}$ \\
\hline $\begin{array}{l}\text { 13-Amors dont } \\
\text { sui espris }\end{array}$ & 4 & $\begin{array}{l}\text { Ré authente } \\
\text { finissant en } \\
\text { plagal }\end{array}$ & $9 \mathrm{M}$ & $\begin{array}{l}\text { ABABCDEFC'DG } \\
\text { ABABCDECDDB } \\
\text { ABABCDECDEF } \\
\text { ababcdefghi } \\
\end{array}$ & $\begin{array}{l}\text { Version 1: } \\
\text { contrafactum de } \\
\text { R } 1545\end{array}$ \\
\hline $\begin{array}{l}14-D \text { 'une amor } \\
\text { coie et serie }\end{array}$ & 1 & Sol authente & $9 \mathrm{M}$ & ABABCDEFA'G & $\begin{array}{l}\text { Contrafactum de } \\
\text { R } 1356\end{array}$ \\
\hline $\begin{array}{l}\text { 16-Ja pour yver } \\
\text { pour noif ne } \\
\text { pour gelee }\end{array}$ & 1 & Sol plagal & \begin{tabular}{|l|}
$7 \mathrm{~m}$ \\
étendue à \\
$8 \mathrm{j}$ \\
\end{tabular} & $\begin{array}{l}\text { ABABCDEF + } \\
\text { refrain variable } \\
\text { selon les strophes }\end{array}$ & $\begin{array}{l}\text { Contrafactum de } \\
\text { R } 1495\end{array}$ \\
\hline
\end{tabular}

Trois des chansons dont s'est inspiré Gautier de Coinci, R 482, Bien doi chanter cui fine Amours adrece, R 1495, Li plus se plaint d'Amours, mais je n'os dire et R 1545, Amours dont sui espris, sont attribuées à Blondel de Nesle. R 2030, De chanter me semont amours, est attribuée à Vielart de Corbie. R 1429, Chanter me fet ce dont je crien morir est attribuée à Pierre de Molins. R 1356, Je chant, c'est mout mauvais signes, est attribuée à Gilles de Maisons ${ }^{3}$. L'activité créatrice du premier se place entre 1175 environ et 1200-1210. Gilles de Maisons, que l'on identifie généralement avec Gilles de Vieux-Maisons en est contemporain, quoique probablement légèrement plus tardif. Ami des deux précédents, Pierre de Molins a dû composer les trois seules chansons qu'on lui connaisse entre la fin du XII ${ }^{\mathrm{e}}$ siècle et les années 1210-1214. Quant à Vielart de Corbie, trouvère également peu prolixe (deux chansons seulement lui sont attribuées avec certitude), il est presque inconnu ${ }^{4}$. Les sources profanes des chansons de Gautier, toutes contemporaines à quelques années près les unes des autres, nous ramènent donc aux dernières années du XII siècle ou aux premières du $\mathrm{XIII}^{\mathrm{e}}$, soit entre quarante et quinze ans avant leur utilisation dans les Miracles.

Je n'ai retrouvé aucun contrafactum parmi les chansons insérées dans Guillaume de Dole. Le roman fournit cependant de précieuses indications quant aux poètes lyriques cités, la plupart d'entre eux étant expressément mentionnés par Jean Renart:

\footnotetext{
${ }^{3}$ Jacques Chailley étudie précisément les sources de Gautier de Coinci dans son étude des Miracles : Jacques CHAILLEY, Les chansons de Gautier de Coinci, Edition critique d'après le ms. de Soissons (réputé perdu), Thèse complémentaire présentée à la Faculté des Lettres de Paris, 1952, 300 p.

${ }^{4}$ Holger Petersen-Dyggve, Onomastique des trouvères, Helsinki, 1934 (Annales Academia Scientiarum Fennica, B, XXX, I), p. 249.
} 
Anne IBOS-AUGÉ

\begin{tabular}{|c|c|c|c|c|c|}
\hline $\begin{array}{c}\mathrm{N}^{\circ} \text { d'ordre dans } \\
\text { l'œuvre et } \\
\text { incipit }\end{array}$ & $\begin{array}{l}\mathrm{N}^{\circ} \text { de } \\
\text { répertoire } \\
\text { biblio. }\end{array}$ & $\begin{array}{l}\text { Attribution } \\
\text { donnée dans } \\
\text { les } \mathrm{mss}^{5}\end{array}$ & $\begin{array}{c}\text { Attribution } \\
\text { donnée } \\
\text { dans } \\
\text { l'œuvre } \\
\end{array}$ & $\begin{array}{l}\text { Position } \\
\text { et } \\
\text { chanteur }\end{array}$ & $\begin{array}{c}\text { Autres } \\
\text { localisations }\end{array}$ \\
\hline $\begin{array}{l}\text { 11-Quant flors et } \\
\text { glais et verdure } \\
\text { s'esloigne }\end{array}$ & $\begin{array}{l}\text { R } 1779= \\
2119\end{array}$ & $\begin{array}{l}\text { MKNXPC: } \\
\text { Gace Brulé }\end{array}$ & Gace Brulé & $\begin{array}{l}\text { V. } 846 \\
\text { Conrad et } \\
\text { Juglet }\end{array}$ & $\begin{array}{l}\text { MKNXPVORLU } \\
C\end{array}$ \\
\hline $\begin{array}{l}\text { 12-Li nouviaus tens } \\
\text { et mais [et violete] }\end{array}$ & $\begin{array}{l}\text { R } 985= \\
986\end{array}$ & $\begin{array}{l}\text { MTaAKXP : } \\
\text { Châtelain de } \\
\text { Couci } \\
\end{array}$ & Sans & $\begin{array}{l}\text { V. } 923 \\
\text { Conrad }\end{array}$ & $\begin{array}{l}\text { MTaAKXPVLO } \\
\text { RUC } \\
\text { Couci } \\
\end{array}$ \\
\hline $\begin{array}{l}\text { 16-Lors que li jor } \\
\text { sont lonc en mai }\end{array}$ & $\begin{array}{l}\text { PC } 262 n^{\circ} \\
2\end{array}$ & $\begin{array}{l}\text { ABCDEJKMR } \\
\text { SSgA } A^{1} \text { : } \\
\text { Jaufré Rudel } \\
\text { W : Gaucelm } \\
\text { Faidit } \\
\end{array}$ & Sans & $\begin{array}{l}\text { V. } 1301 \\
\text { Nicole }\end{array}$ & $\begin{array}{l}\text { Mss Tbs : } \\
A B C D E J K M \mathrm{R} S S \\
g A^{l} e \mathrm{WX}\end{array}$ \\
\hline $\begin{array}{l}\text { 17-Dés que Fromonz } \\
\text { au Veneor tença }\end{array}$ & & & $\begin{array}{l}\text { Geste de } \\
\text { Gerbert de } \\
\text { Metz }\end{array}$ & $\begin{array}{l}\text { V. } 1335 \\
\text { Un } \\
\text { jongleur, } \\
\text { ou sa } \\
\text { sœur } \\
\end{array}$ & \\
\hline $\begin{array}{l}\text { 18-Loial amor qui en } \\
\text { fins cuer s'est mise }\end{array}$ & R 1635 & & $\begin{array}{l}\text { Renaut de } \\
\text { Beaujeu }\end{array}$ & $\begin{array}{l}\text { V. } 1456 \\
\text { Conrad } \\
\end{array}$ & OUC \\
\hline $\begin{array}{l}\text { 22-Contre le tens } \\
\text { que voi frimer }\end{array}$ & $\begin{array}{l}\text { R } 857= \\
2027\end{array}$ & $\begin{array}{l}\text { MTKNX : } \\
\text { Gace Brulé } \\
\mathrm{P}: \text { Gautier de } \\
\text { Dargies }\end{array}$ & Sans & $\begin{array}{l}\text { V. } 2027 \\
\text { Juglet }\end{array}$ & MTKNXPVOLR \\
\hline $\begin{array}{l}\text { 34-Je di que c'est } \\
\text { granz folie }\end{array}$ & $\begin{array}{l}\text { R } 1232 \\
\text { (3ème st.) }\end{array}$ & $\begin{array}{l}\text { C: Gace Brulé } \\
\mathrm{T}: \text { Aubuin de } \\
\text { Sezanne } \\
M: \text { Pierre de } \\
\text { Beaumar- } \\
\text { chais } \\
\end{array}$ & Gace Brulé & $\begin{array}{l}\text { V. } 3625 \\
\text { Conrad }\end{array}$ & $\begin{array}{l}\frac{\text { Violette }}{\text { MTUC }} \\
\text { Ms. Tbs } Q\end{array}$ \\
\hline $\begin{array}{l}\text { 35-Por quel forfet ne } \\
\text { por quel ochoison }\end{array}$ & $\begin{array}{l}\text { R } 1876 a= \\
1872= \\
1884\end{array}$ & $\begin{array}{l}\text { KXP : } \\
\text { Châtelain de } \\
\text { Couci } \\
\text { MTR : Roger } \\
\text { d'Andeli } \\
\text { C: Gace } \\
\text { Brulé } \\
\end{array}$ & Sans & $\begin{array}{l}\text { V. } 3751 \\
\text { Conrad }\end{array}$ & $\begin{array}{l}\text { MTKXPVORUC } \\
H Z a G\end{array}$ \\
\hline $\begin{array}{l}\text { 36-Ja de chanter en } \\
\text { ma vie }\end{array}$ & R 1229 & $\begin{array}{l}\mathrm{M}: \text { Gace } \\
\text { Brulé } \\
\mathrm{a}: \text { Blondel de } \\
\text { Nesle }\end{array}$ & $\begin{array}{l}\text { Renaut de } \\
\text { Sablœil }\end{array}$ & $\begin{array}{l}\text { V. } 3883 \\
\text { Conrad }\end{array}$ & $\mathrm{MaOCKNXPH}$ \\
\hline
\end{tabular}

\footnotetext{
${ }^{5}$ Les attributions douteuses, dans ce tableau et les suivants, sont signalées par l'emploi de caractères italiques. Pour différencier les noms de trouvères des titres de romans (dans le cas du châtelain de Couci par exemple), ces derniers sont écrits en caractères italiques et soulignés.

${ }^{6}$ Dans ce tableau et les suivants, les sigles des manuscrits sont notés en caractères italiques quand ils comportent des versions non musicales des chansons concernées.
} 


\begin{tabular}{|c|c|c|c|c|c|}
\hline $\begin{array}{l}\text { 37-Quant li dous } \\
\text { tens et la sesons } \\
\text { s'asseüre }\end{array}$ & R 2086 & $\begin{array}{l}\text { MTaKNX : } \\
\text { Vidame de } \\
\text { Chartres } \\
\mathrm{R}: \text { Châtelain } \\
\text { de Couci } \\
\text { C: Gace } \\
\text { Brulé }\end{array}$ & $\begin{array}{l}\text { Vidame de } \\
\text { Chartres }\end{array}$ & $\begin{array}{l}\text { V. } 4127 \\
\text { Un jeune } \\
\text { homme }\end{array}$ & $\begin{array}{l}\text { MTaKNXPVOR } \\
\text { BUC }\end{array}$ \\
\hline $\begin{array}{l}\text { 41-Bele m'est la voiz } \\
\text { altane }\end{array}$ & $\begin{array}{l}\mathrm{PC} 124 \mathrm{n}^{\circ} \\
5\end{array}$ & $\begin{array}{l}C: \text { Daude de } \\
\text { Pradas }\end{array}$ & Sans & $\begin{array}{l}\text { V. } 4653 \\
\text { Un } \\
\text { chanteur } \\
\text { non } \\
\text { précisé }\end{array}$ & Mss Tbs WC \\
\hline $\begin{array}{l}\text { 45-Quan voi l'aloete } \\
\text { moder }\end{array}$ & $\begin{array}{l}\text { PC } 70 n^{\circ} \\
43\end{array}$ & $\begin{array}{l}\frac{\text { Breviari }}{\text { d'amor }} \\
\underline{\text { Razos de }} \\
\begin{array}{l}\text { trobar } \\
\text { So fo el }\end{array} \\
\text { temps... } \\
\text { ACDEFGIKK } \\
\text { LMNPQRSUV } \\
a: \text { Bernart de } \\
\text { Ventadorn } \\
N^{2}: \text { Peire } \\
\text { Vidal } \\
\end{array}$ & Sans & $\begin{array}{l}\text { V. } 5212 \\
\text { Un } \\
\text { chevalier }\end{array}$ & $\begin{array}{l}\frac{\text { Breviari d'amor }}{\text { Razos de trobar }} \\
\text { So fo el temps... } \\
\text { Violette } \\
\text { Mss tbs : } \\
\text { ACDEFGIKK }{ }^{a} L \\
M N P Q \mathrm{R} S U V a \mathrm{~W} \\
O \\
\text { VeAgXN }\end{array}$ \\
\hline $\begin{array}{l}\text { 46-Lors que florist la } \\
\text { bruiere }\end{array}$ & R 1322a & $\begin{array}{l}M i \text { : Gontier } \\
\text { de Soignies }\end{array}$ & $\begin{array}{l}\text { Gontier de } \\
\text { Soignies }\end{array}$ & $\begin{array}{l}\text { V. } 5232 \\
\text { Un jeune } \\
\text { homme }\end{array}$ & $M i$ \\
\hline
\end{tabular}

Mêlant les inspirations du sud et du nord, les chansons choisies par Jean Renart traversent par là-même un temps plus long que ne le faisaient les sources profanes empruntées par Gautier de Coinci pour ses Miracles: contemporains, Jaufré Rudel et Bernart de Ventadorn sont actifs au milieu du XII ${ }^{\mathrm{e}}$ siècle. La laisse de Gerbert de Metz pose un problème, tout au moins au lecteur contemporain : elle est en effet absente des versions conservées de la chanson de geste, datable quant à elle entre 1185 environ et... les années de composition de Guillaume de Dole ${ }^{7}$. Cette absence peut avoir deux explications : il peut s'agir d'une version ou d'une variante disparue ; ou bien Jean Renart peut l'avoir lui-même écrite. Cette dernière possibilité me paraît tout à fait plausible pour plusieurs raisons. D'abord, les liens entre la laisse et la narration sont multiples, la première empruntant à la seconde des éléments précis. Il y est question d'un heaume détruit:

A Geronvile, au pié dou pont de ça,

uns chevaliers un tel cop m'i dona

desor mon heaume que tot le m'enbarra ${ }^{8}$;

${ }^{7}$ On consultera à propos de la geste l'article de Félix Lecoy, «Sur Gerbert de Metz: lieux et date », Romania, 77, 1956, p. 417-435.

${ }^{8}$ Jean Renart, Le roman de la rose ou de Guillaume de Dole, éd. par Félix Lecoy, Paris, Champion, 1979, v. 1360-1362. 
or Guillaume brise le sien lors de la joute de Saint-Trond, comme il l'avoue à Jouglet :

\author{
Il ne me faut q'un tot seul heaume (... $)^{9}$ \\ Je perdi l'autre jour le mien \\ quant je fui pris a Rougemont ${ }^{10}$;
}

le don du cheval évoqué dans le fragment épique rappelle les dons faits par Guillaume à Jouglet, puis par Conrad à Guillaume (parmi lesquels on compte un cheval, comme dans la laisse); on peut aussi y lire un parallèle entre la preuve difficile à fournir, de la captivité de Foucon et Rocelin dans la laisse comme de la culpabilité ou de l'innocence de Lienor dans le roman, ainsi qu'un jeu de symétrie des couples Guiret/son fils et Nicole/le sénéchal, tous ces éléments contribuant à relier le microcosme de l'intermède épique au macrocosme que représente le roman. La seconde raison possible à la création du fragment épique par l'auteur du roman se trouve dans le prologue même du Guillaume de Dole, affirmant :

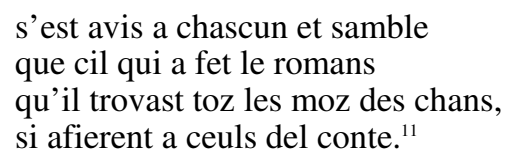

Si Jean Renart était si fier de la parfaite concordance entre la trame narrative de son œuvre et les chansons qu'il y avait insérées, il est bien probable que quelques-unes des insertions aient pu ne pas seulement «sembler» être écrites par lui. Quoiqu'il en soit, le seul fait que Jean Renart ait voulu témoigner de la connaissance qu'il avait de la geste, qu'il ait ou non composé cette laisse, donne la mesure de la mémoire et de la référence temporelle qu'il désirait laisser à ses lecteurs. Ses autres références se rapportent également pour la plupart aux vingt dernières années du XII ${ }^{\mathrm{e}}$ siècle. Quelques questions se posent quant aux attributions données dans la narration, quand d'autres sont proposées par différents témoins. Le roman lui-même peut néanmoins parfois aider à prendre parti. La chanson 34, dont l'attribution à Gace est confirmée par le chansonnier $C$, est bien probablement du trouvère champenois. La chanson 36 est considérée comme douteuse à la fois par les éditeurs de Gace $^{12}$ et de Blondel $^{13}$ : rien ne s'oppose donc à ce qu'elle soit de Renaut de Sablœil, dont on ignore en revanche presque tout. Selon Holger PetersenDyggve $^{14}$, il s'agirait en réalité de Robert IV de Sablœil, croisé en 1189 avec Richard

\footnotetext{
${ }^{9}$ Ibid., v. 1650.

${ }^{10}$ Ibid., v. 1658-1659.

${ }^{11}$ Ibid., v. 26-29.

${ }^{12}$ Holger Petersen Dyggve, Gace Brulé, trouvère champenois. Édition des chansons et étude historique, Helsinki, 1951 (Mémoires de la Société néophilologique d'Helsinki, 16).

${ }^{13}$ Leo Wiese, Die Lieder des Blondels de Nesle, Dresde, 1904 (Gesellschaft für romanische Literatur, 5).

${ }^{14}$ Holger Petersen Dyggve, «Personnages historiques figurant dans la poésie lyrique française des XII ${ }^{\mathrm{e}}$ et XIII ${ }^{\mathrm{e}}$ siècles. XX. Renaut de Sablœil et la comtesse de Meulant», Neuphilologische Mitteilungen, 45, 1944, p. 61-91.
} 
Cœur de Lion, puis grand maître du Temple jusqu'à sa mort en 1196. Il aurait écrit cette chanson avant son départ pour la croisade. Quant à la chanson 42, disparue du chansonnier $M$, elle se trouvait dans la table du manuscrit avec le nom de Gontier, ce qui confirme l'attribution donnée par Jean Renart. Une dernière interrogation est soulevée par la «chançon auvrignace» des vers 4653-4659. Elle est attribuée par le seul chansonnier de troubadours $C$ à Daude de Pradas. Or si la célébrité de ce troubadour - cité par Matfre Ermengaud dans son Breviari d'amor parmi les meilleurs de son temps - justifie sa place dans le roman aux côtés des chansons les plus connues de ses compatriotes de langue d'oc, son «temps» l'écarte singulièrement des autres références lyriques du roman: il en serait le seul poète exactement contemporain. Un des derniers troubadours, il serait né vers 1194 et aurait disparu après 1282. Toutefois, les débuts de son activité poétique - autour de 1215 - permettent bien que Jean Renart ait eu connaissance de ses textes et ait choisi d'en citer un dans son roman. Ceci représenterait d'ailleurs un argument supplémentaire en faveur de la datation du Guillaume de Dole vers 1228, et non avant, comme le pensaient les premiers éditeurs du roman ${ }^{15}$.

L'histoire musicale du Guillaume de Dole se place donc sur une période relativement longue, de la mi-XII ${ }^{\mathrm{e}}$ aux années précédant directement le roman. Elle s'inscrit également dans une géographie assez vaste, incluant la langue d'oc, non dans sa forme primitive, mais dans une sorte de traduction-adaptation procédant de la recréation poétique. Il semble que, familier de la poésie et de la langue occitanes, Jean Renart ait désiré faciliter la tâche de ses lecteurs tout en conservant l'essentiel des sonorités de la langue qu'il empruntait et se réappropriait, à moins qu'il ne se soit contenté de réutiliser une forme linguistique courante en France du nord, adaptation orale de la langue d'oc. ${ }^{16}$

Gerbert de Montreuil ne cite plus ses sources poétiques, mais la plupart des chansons sont l'œuvre de poètes connus par ailleurs :

Voir tableau page suivante

\footnotetext{
${ }^{15}$ Rita Lejeune, qui le datait des années 1212-1213, et surtout G. Servois, qui l'affirmait écrit entre 1199 au plus tôt et 1201 au plus tard.

${ }^{16}$ Voir à ce propos l'article de Fabienne Gégou, «Jean Renart et la lyrique occitane », Mélanges de langue et de littérature médiévales offerts à Pierre Le Gentil, Paris, SEDES et CDU réunis, p. 319-323.
} 


\begin{tabular}{|c|c|c|c|c|c|}
\hline $\begin{array}{c}\mathrm{N}^{\circ} \text { d'ordre dans } \\
\text { l'œuvre et } \\
\text { incipit }\end{array}$ & $\begin{array}{l}\mathrm{N}^{\circ} \text { de } \\
\text { répertoire } \\
\text { biblio. }\end{array}$ & $\begin{array}{c}\text { Attribution } \\
\text { donnée dans les } \\
\text { mss }\end{array}$ & Position & Chanteur & $\begin{array}{l}\text { Autres } \\
\text { localisations }\end{array}$ \\
\hline $\begin{array}{l}\text { 8-Quant biele } \\
\text { dame et fine } \\
\text { amors m'en prie }\end{array}$ & R 1198 & $\begin{array}{l}\text { MTKNXP : Gace } \\
\text { Brulé }\end{array}$ & $\begin{array}{l}\text { V. 191- } \\
198\end{array}$ & Gerart & $\begin{array}{l}\text { MTKNXPV } \\
\text { OL }\end{array}$ \\
\hline $\begin{array}{l}\text { 11-Il n'est anuis } \\
\text { ne faillemens }[A]\end{array}$ & $\begin{array}{l}\text { PC, 70, } 1 \\
\text { (3ème st.) }\end{array}$ & $\begin{array}{l}\text { Breviari d'amor } \\
\text { So fo el temps... } \\
\text { Razos de trobar } \\
\text { ABCDD } F \mathrm{G} I K L M \\
P Q \mathrm{R} S T U V a: \\
\text { Bernart de } \\
\text { Ventadorn } \\
N^{2}: \text { Berenguier de } \\
\text { Noya } \\
\end{array}$ & $\begin{array}{l}\text { V. 324- } \\
331\end{array}$ & Euriaut & $\begin{array}{l}\frac{\text { Breviari d'amor }}{\text { So fo el temps... }} \\
\text { Razos de trobar } \\
\text { Mss tbs : } \\
\text { ABCDD FGIKLM } \\
\text { PQRSTUVVeAg } \\
\text { WXa N } N^{2}\end{array}$ \\
\hline $\begin{array}{l}12-\text { Amors mi font } \\
\text { renvoisier et } \\
\text { canter }\end{array}$ & $\begin{array}{l}\text { R } 810= \\
796\end{array}$ & $\begin{array}{l}\text { MTa : Moniot } \\
\text { d'Arras }\end{array}$ & $\begin{array}{l}\text { V. } 441- \\
449\end{array}$ & Euriaut & $\mathrm{M} T a$ \\
\hline $\begin{array}{l}\text { 15-Cil qui } \\
\text { d'amours me } \\
\text { conseille }\end{array}$ & $\begin{array}{l}\text { R 565= } \\
567\end{array}$ & $\begin{array}{l}\text { MKNXPC : Gace } \\
\text { Brulé }\end{array}$ & $\begin{array}{l}\text { V. 1266- } \\
1275\end{array}$ & $\begin{array}{l}\text { Le duc de } \\
\text { Metz }\end{array}$ & $\begin{array}{l}\text { Meliacin } \\
\text { MKNXPVORHLU } \\
C G \\
\end{array}$ \\
\hline $\begin{array}{l}\text { 16-Par Diu! Je } \\
\text { tienc a folie }\end{array}$ & $\begin{array}{l}\text { R } 1232 \\
\text { (3ème st.) }\end{array}$ & $\begin{array}{l}\text { CGuillaume de } \\
\text { Dole : Gace Brulé } \\
M: \text { Pierre de } \\
\text { Beaumarchais } \\
\mathrm{T}: \text { Aubuin de } \\
\text { Sezanne }\end{array}$ & $\begin{array}{l}\text { V. 1315- } \\
1321\end{array}$ & Gerart & $\begin{array}{l}\text { Guillaume de Dole } \\
\text { MTUC } \\
\text { Ms tbs } Q\end{array}$ \\
\hline $\begin{array}{l}\text { 17-Grans fu la } \\
\text { cours en la sale a } \\
\text { Loon }\end{array}$ & & & $\begin{array}{l}\text { V. } 1407- \\
1428\end{array}$ & Gerart & $\underline{\text { Aliscans }}$ \\
\hline $\begin{array}{l}\text { 23-Destrois, } \\
\text { pensis, en esmai }\end{array}$ & R 77 & Audefroi le Bâtard & \begin{tabular}{|l} 
V. 3236- \\
3243 \\
\end{tabular} & Gerart & MT \\
\hline $\begin{array}{l}\text { 26-Par un seul } \\
\text { baisier de cuer a } \\
\text { loisir }\end{array}$ & $\begin{array}{l}\text { R } 1405 \\
\text { (4ème st.) }\end{array}$ & $\begin{array}{l}\text { MTaAC: } \\
\text { Guillaume le } \\
\text { Vinier } \\
Y: \text { Gilles le Vinier }\end{array}$ & $\begin{array}{l}\text { V. 3641- } \\
3647\end{array}$ & Gerart & $\mathrm{MTa} A C Y$ \\
\hline $\begin{array}{l}\text { 29-Quan voi la } \\
\text { loete moder }\end{array}$ & $\begin{array}{l}\text { PC, 70, } \\
43\end{array}$ & $\begin{array}{l}\text { Breviari d'amor } \\
\text { Razos de trobar } \\
\text { So fo el temps... } \\
\text { ACDEFGIKK } L M \\
N P Q R S U V a: \\
\text { Bernart de } \\
\text { Ventadorn } \\
N^{2}: \text { Peire Vidal } \\
\end{array}$ & $\begin{array}{l}\text { V. 4187- } \\
4195\end{array}$ & Gerart & $\begin{array}{l}\text { Breviari d'amor } \\
\text { Razos de trobar } \\
\text { So fo el temps... } \\
\text { Guillaume de Dole } \\
\text { Mss tbs : } \\
\text { ACDEFGIKK }{ }^{a} L M \\
N P Q R S U V a \mathrm{~W} O V e \\
\text { AgXN }\end{array}$ \\
\hline $\begin{array}{l}\text { 33-Par Diu, } \\
\text { Amours, grief } \\
\text { m'est a consirrer }\end{array}$ & $\begin{array}{l}\text { R } 679 \\
\text { (3ème st.) }\end{array}$ & $\begin{array}{l}\text { MTAXP Couci } \\
\text { Vergi : Châtelain } \\
\text { de Couci }\end{array}$ & $\begin{array}{l}\text { V. 4624- } \\
4631\end{array}$ & Gerart & \begin{tabular}{|l} 
Couci \\
Vergi \\
MTAKXPVORUC
\end{tabular} \\
\hline $\begin{array}{l}\text { 39-Ne mi sont pas } \\
\text { ochoison de } \\
\text { canter }\end{array}$ & R 787 & $\begin{array}{l}\text { KNXPC: Gace } \\
\text { Brulé } \\
\text { a : Guillaume le } \\
\text { Vinier } \\
\end{array}$ & $\begin{array}{l}\text { V. 5789- } \\
5797\end{array}$ & Gerart & $\begin{array}{l}\text { aKNXPVOL } H Z a \mathrm{U} \\
C\end{array}$ \\
\hline $\begin{array}{l}\text { 42-Qui bien } \\
\text { aimme ne se doit } \\
\text { esmaier }\end{array}$ & $\begin{array}{l}\text { R } 1259= \\
1318 \\
\text { (2ème st.) }\end{array}$ & $\begin{array}{l}\text { MT : Moniot } \\
\text { d'Arras }\end{array}$ & $\begin{array}{l}\text { V. 6614- } \\
6620\end{array}$ & Gerart & MTU \\
\hline
\end{tabular}


Les partis pris sont ici très différents. Certes, on trouve encore dans le roman de Gerbert de Montreuil des trouvères de la première génération en la personne du châtelain de Couci et de Gace Brulé, avec une nette préférence pour ce dernier. Les «sons poitevins » (ainsi présentés par l'auteur, sauf en ce qui concerne la chanson 29, qualifiée de son «provençal » dans les manuscrits $B$ et $C$ ) sont moins diversifiés que chez son prédécesseur : seul Bernart de Ventadorn a été choisi; les trois autres versions de l'insertion 11 sont soit des chansons non identifiées (mss $B D$ ), soit la traduction de l'une d'entre elles (ms. $C$ ). Le fragment épique ne soulève ici aucune question : il s'agit d'une laisse d'Aliscans qui, pour sa part, nous renvoie à l'extrême fin du siècle précédant le roman. La nouveauté, toutefois, consiste en l'introduction de chansons de trouvères contemporains de la Violette, ce qui n'était qu'une seule fois le cas chez Jean Renart. Moniot d'Arras, deux fois cité - même si le refrain de la chanson 12 a été modifié par Gerbert de Montreuil par rapport à l'original prévu par le trouvère - est actif entre 1213 et 1239 environ. On date généralement les débuts poétiques de son ami et concitoyen Guillaume le Vinier (mort en 1245) des années 1220. Quant à Audefroi le Bâtard, autre arrageois, on situe son activité littéraire dans le premier tiers du $\mathrm{XIII}^{\mathrm{e}}$ siècle, ce qui en fait un troisième contemporain du roman. Si l'on ajoute à ces derniers Renaut de Trie, né autour de 1195 et actif dans la première moitié du XIII ${ }^{\mathrm{e}}$ siècle, auteur de la chanson $\mathrm{R} 863$ Bien doit Amours guerredonner qui a probablement servi de modèle à l'insertion 34 Lasse, comment porai durer, le nombre de trouvères cités contemporains du roman se porte à quatre, ce qui dénote bien une évolution par rapport aux choix de Jean Renart. Si Gerbert de Montreuil cite volontiers des trouveurs dont la renommée précède l'époque de son roman, il fait également, à part égale, œuvre de témoin direct de son propre temps musical, ce que ne faisait que peu son modèle.

Tous les modèles profanes des chansons du Ludus ont été notés, peut-être par l'auteur lui-même, dont l'éditeur du roman ${ }^{17}$ pense qu'il en fut également le copiste, sur l'unique manuscrit conservant le roman :

\begin{tabular}{|l|l|l|l|l|l|}
\hline $\begin{array}{c}\mathrm{N}^{\circ} \text { d'ordre dans l'œuvre } \\
\text { et incipit } \\
\text { Incipit du contrafactum }\end{array}$ & $\begin{array}{c}\mathrm{N}^{\circ} \mathrm{de} \\
\text { répertoire } \\
\text { biblio. }\end{array}$ & $\begin{array}{l}\text { Attribution } \\
\text { donnée dans } \\
\text { les mss }\end{array}$ & Position & Chanteur & $\begin{array}{l}\text { Localisations } \\
\text { du } \\
\text { contrafactum }\end{array}$ \\
\hline $\begin{array}{l}\text { 1-O quam fallax est } \\
\text { mundi gloria } \\
\text { Quant voi paroir la } \\
\text { fueille en la ramee }\end{array}$ & R 550 & $\begin{array}{l}\text { MT : Sauvage } \\
\text { de Bethune } \\
\text { KNXPC: } \\
\text { Gace Brulé }\end{array}$ & $\S 37$ & Musique & $\begin{array}{l}\text { MTKNXPVO } \\
\text { LC }\end{array}$ \\
\hline $\begin{array}{l}\text { 2-Ave gemma qui lucis } \\
\text { copia } \\
\text { Tant ai Amours servies } \\
\text { longuement }\end{array}$ & $\mathrm{R} 711$ & $\begin{array}{l}\text { MaKNXPR : } \\
\text { Roi de } \\
\text { Navarre }\end{array}$ & $\S 67,1$ & $\begin{array}{l}\text { Le poète : } \\
\text { invocation } \\
\text { entendue } \\
\text { par } \\
\text { Prudence } \\
\text { aux cieux }\end{array}$ & $\begin{array}{l}\text { MtaAKNXPV } \\
\text { ORS } \text { Paris, Bibl. } \\
\text { Mazarine 753 }\end{array}$ \\
\hline
\end{tabular}

${ }^{17}$ Adam de la Bassée, Ludus super Anticlaudianum, éd. par Paul Bayart, Tourcoing, Georges Frère, $1930, \mathrm{CVI} / 340 \mathrm{p}$. 


\begin{tabular}{|c|c|c|c|c|c|}
\hline $\begin{array}{l}\text { 12-O constantiae } \\
\text { dignitas } \\
\text { Quant voi la glaie meüre }\end{array}$ & R 2107 & $\begin{array}{l}\text { aKXPF : } \\
\text { Raoul de } \\
\text { Soissons } \\
\mathrm{N}: \text { Pierre de } \\
\text { Soissons } \\
C \text { : Perrin } \\
\text { d'Angicourt }\end{array}$ & $\S 79$ & $\begin{array}{l}\text { Le poète : } \\
\text { invocation } \\
\text { entendue } \\
\text { par } \\
\text { Prudence } \\
\text { aux cieux }\end{array}$ & $\begin{array}{l}\text { aKNXPVRSF } \\
\text { UC } \\
\text { Metz, BM } 535 \\
\text { Paris, Bibl. } \\
\text { Mazarine } 54\end{array}$ \\
\hline $\begin{array}{l}\text { 15-Ave princeps celestis } \\
\text { curie } \\
\text { Loiaus desir et pensee } \\
\text { jolie }\end{array}$ & R 1172 & $\begin{array}{l}\text { a } C: \text { Martin le } \\
\text { Beguin de } \\
\text { Cambrai }\end{array}$ & $\S 83,1-2$ & $\begin{array}{l}\text { Le poète: } \\
\text { invocation } \\
\text { entendue } \\
\text { par } \\
\text { Prudence } \\
\text { aux cieux }\end{array}$ & $\begin{array}{l}\text { aVORIUC } \\
\text { Meliacin } \\
\end{array}$ \\
\hline $\begin{array}{l}\text { 17-Ave, rosa rubens et } \\
\text { tenera } \\
\text { Tant ai d'amours apris } \\
\text { et entendu }\end{array}$ & R 2054 & $\begin{array}{l}C: \text { Gadifer } \\
\text { d'Avion }\end{array}$ & $\S 85$ & $\begin{array}{l}\text { Le poète : } \\
\text { invocation } \\
\text { entendue } \\
\text { par } \\
\text { Prudence } \\
\text { aux cieux }\end{array}$ & $\mathrm{VOUC}$ \\
\hline $\begin{array}{l}\text { 29-Felix qui humilium } \\
\text { L'autrier estoie montés }\end{array}$ & R 936 & $\begin{array}{l}\text { KNXP : Duc } \\
\text { de Brabant }\end{array}$ & $\$ 129$ & Humilité & KNXPV \\
\hline
\end{tabular}

Il convient d'ajouter à ces modèles le motet M 361 Hé, (Dieus) quant je remir son cors le gai/Amor, à l'origine de l'insertion 32 O quam solemnis legatio. Ce motet est anonyme mais sa localisation dans le corpus le plus ancien du chansonnier de Montpellier permet d'en avancer le terminus ante quem: 1220. L'insertion suivante $\left(\mathrm{n}^{\circ}\right.$ 33: O lampas) porte sur le manuscrit la mention: Respons[ori]um de Job super illud O lampas eccelesie de sancta Elizabeth. La canonisation d'Élisabeth datant de 1235 , la rubrique ne se justifie que si le répons est effectivement postérieur à cette date. En ce qui concerne les trouvères identifiés, la datation couvre une période allant des années 1220 (commencement probable de l'activité poétique de Thibaut de Champagne, né en 1201 et mort en 1253) aux années 1260: l'œuvre poétique de Raoul de Soissons est située entre les années 1235-1240 et 1260, celle du duc de Brabant entre 1250 environ et 1261 , date de sa mort. On ne sait que fort peu de choses de Sauvage de Bethune (à qui doit être rétablie la paternité de l'insertion 1), Martin le Béguin de Cambrai et Gadifer d'Avion, tous trois actifs au milieu du XIII ${ }^{\mathrm{e}}$ siècle. La chanson de ce dernier a également servi de modèle à un trouvère contemporain d'Adam de la Bassée, Lambert Ferri, chanoine d'Arras de 1268 à 1301 pour R 2053 J'ai tant d'Amors apris et entendu. L'histoire musicale des emprunts profanes d'Adam de la Bassée commence donc plus de soixante ans avant l'écriture de son roman (autour de 1280) pour s'achever au plus tard une vingtaine d'années avant ${ }^{18}$.

Girart d'Amiens, quand il écrit Meliacin dans les années 1285-1288, ne cite pas ses sources poétiques. Neuf d'entre elles sont néanmoins connues :

${ }^{18}$ On pourra consulter à ce sujet l'article du musicologue Andrew Hughes, « The Ludus super Anticlaudianum of Adam de la Bassée », Journal of the American Musicological Society, 23, 1970, p. 1-25. 


\begin{tabular}{|c|c|c|c|c|c|}
\hline $\begin{array}{c}\mathrm{N}^{\circ} \text { d'ordre } \\
\text { dans l'œuvre } \\
\text { et incipit }\end{array}$ & $\begin{array}{l}\mathrm{N}^{\circ} \mathrm{de} \\
\text { répertoire } \\
\text { biblio. }\end{array}$ & $\begin{array}{c}\text { Attribution } \\
\text { donnée dans } \\
\text { les mss }\end{array}$ & Position & Chanteur & $\begin{array}{c}\text { Autres } \\
\text { localisations }\end{array}$ \\
\hline $\begin{array}{l}\text { 2-Cil qui d'Amours me } \\
\text { conseille }\end{array}$ & R $565=567$ & $\begin{array}{l}\text { MKNXPC: } \\
\text { Gace Brulé }\end{array}$ & V. 3598 & Meliacin & $\begin{array}{l}\frac{\text { Violette }}{\text { MKNXPVOR }} \\
\text { HLUCG }\end{array}$ \\
\hline $\begin{array}{l}\text { 3-Lors quant je voi le } \\
\text { buisson en verdure }\end{array}$ & R 2118 & $\begin{array}{l}\text { aKN : Perrin } \\
\text { d'Angicourt }\end{array}$ & V. 3695 & Celinde & aKNVORZ \\
\hline $\begin{array}{l}\text { 9-Bone aventure aviegne } \\
\text { a fol espoir }\end{array}$ & $\begin{array}{l}\text { R 741 = } 991 \\
\text { (3ème st.) }\end{array}$ & $\begin{array}{l}\text { Mta } \\
\text { KNXPR : } \\
\text { Thibaut de } \\
\text { Champagne }\end{array}$ & V. 5794 & Meliacin & $\begin{array}{l}\text { MTaKNXPV } \\
\text { ORSZBUC }\end{array}$ \\
\hline $\begin{array}{l}\text { 12-Desconfortés et de } \\
\text { joie partis }\end{array}$ & R 1073 & $\begin{array}{l}\text { MKNC: } \\
\text { Gautier } \\
\text { d'Épinal } \\
\end{array}$ & V. 8110 & Meliacin & $\begin{array}{l}\text { MTKNPVOR } \\
\text { UCX }\end{array}$ \\
\hline $\begin{array}{l}\text { 15-Puisqu'il m'estuet de } \\
\text { ma dolor chanter }\end{array}$ & R 805 & $\begin{array}{l}\text { MTAR: } \\
\text { Richart de } \\
\text { Fournival } \\
\text { Mi : Roi de } \\
\text { Navarre } \\
\mathrm{N}: \text { Gautier } \\
\text { d'Épinal }\end{array}$ & V. 9027 & Meliacin & MTARNXO \\
\hline $\begin{array}{l}\text { 16-Bone Amors m'a a } \\
\text { son service mis }\end{array}$ & R 1569 & $\begin{array}{l}\mathrm{R} \text { : Girardin } \\
\text { de Boulogne }\end{array}$ & V. 10855 & Meliacin & ORIU \\
\hline $\begin{array}{l}\text { 19-Loiaus desirs et } \\
\text { pensee jolie }\end{array}$ & R 1172 & $\begin{array}{l}\text { aC: Martin le } \\
\text { Beguin de } \\
\text { Cambrai }\end{array}$ & V. 16745 & Meliacin & $\begin{array}{l}\text { aVORIUC } \\
\text { Ludus }\end{array}$ \\
\hline $\begin{array}{l}\text { 20-Li plusor ont } \\
\text { d'Amors chanté }\end{array}$ & R 413 & $\begin{array}{l}\text { MTaKNXP } \\
C \text { : Gace } \\
\text { Brulé } \\
\mathrm{R}: \text { Châtelain } \\
\text { de Couci }\end{array}$ & V. 16823 & Meliacin & $\begin{array}{l}\text { MTaKNXPV } \\
\text { OLRUC }\end{array}$ \\
\hline $\begin{array}{l}\text { 26-Ä̈! amans fins et } \\
\text { vrais }\end{array}$ & R 199 & $\begin{array}{l}\text { MKNPC: } \\
\text { Gautier } \\
\text { d'Épinal }\end{array}$ & V. 17993 & Meliacin & $\begin{array}{l}\text { MKNXPVOR } \\
\text { SBUC }\end{array}$ \\
\hline
\end{tabular}

Gace Brulé, cité à deux reprises, renvoie un siècle avant la composition du roman mais les autres poètes parcourent le XIII ${ }^{\mathrm{e}}$ siècle, de Thibaut de Champagne actif entre les années 20 et 50 à Martin le Beguin de Cambrai, figurant encore en 1276-77 dans les comptes de Guy de Dampierre, en passant par Perrin d'Angicourt et Gautier d'Épinal (disparu vers 1270-1272), dont l'activité poétique et musicale se situe autour de la mi XIII ${ }^{\mathrm{e}}$, ainsi que Girardin de Boulogne, contemporain de Jehan Bretel, et œuvrant donc dans la seconde moitié du siècle. La mémoire musicale profane de Girart d'Amiens est donc la plus longue qu'il nous ait été donné d'observer depuis Gautier de Coinci. Elle est également parmi les plus fidèles, la plus grande partie des insertions du roman (dix-neuf sur vingt-quatre) - y compris les motets, rondeaux et refrains anonymes - se retrouvant par ailleurs. 
Deux chansons du Rosarius sont attribuées par ailleurs à des trouvères connus. L'insertion 2, J'ay un cuer moult lent est, ainsi que l'affirme le compilateur de l'œuvre, de Thibaut d'Amiens et l'insertion 10, Qui bien aime, a tart oublie, est attribuée à Moniot d'Arras. Si ce dernier renvoie à la première moitié du XIII ${ }^{\mathrm{e}}$ siècle, le premier n'est connu que pour la chanson citée dans le Rosarius, également présente dans de nombreux autres manuscrits. Son activité poétique daterait de la fin du $\mathrm{XII}^{\mathrm{e}}$ siècle ou du début du XIII ${ }^{\mathrm{e}}$, sans que l'on puisse à ce jour en préciser davantage l'étendue. Parmi les autres citations du recueil, l'insertion 19, Hé Diex pour quoy n'est bien amez, est un contrafactum de la chanson R 824, Li lousseignols que j'oi chanter, de Pierre le Borgne, originaire de Lille et actif durant la seconde moitié du XIII ${ }^{\mathrm{e}}$ siècle. Enfin, deux autres chansons renvoient à Moniot d'Arras, décidément favori du compilateur: la chanson 3 (reprise en onzième position), $\mathrm{R}$ 2114 De la Virge nete et pure, contrafactum de R 1216 Bone Amour sans tricherie et la chanson 8, R 1183 Toy reclaim, Virge Marie, dont le modèle est R 1135 Amours n'est pas, que c'on die. L'ensemble des références, malheureusement peu nombreuses, couvre donc une période très longue d'un siècle précédant le texte du frère précheur du Soissonnais.

La première référence unique du répertoire des œuvres munies d'insertions lyriques, celle au châtelain de Couci dans le roman éponyme, place volontairement d'emblée son histoire musicale dans les trente dernières années du XII ${ }^{\mathrm{e}}$ siècle, soit un siècle environ avant l'élaboration de la narration. L'erreur d'attribution pour les chansons 1 et 7, toutes deux de Gace Brulé rapproche cette histoire du temps du roman, même si la distance reste grande. La parenté de l'œuvre avec les Vidas de troubadours s'en trouve du même coup renforcée, ces textes ayant été écrits un siècle environ après la période d'activité des poètes qu'ils mettaient en scène.

La référence au même châtelain de Couci dans Vergi, si elle place ce court roman dans le même rapport de dates que celui de Jakemes, se double d'une intention différente, conférant l'historicité au personnage même du roman, qui

Si est en tel point autressi

Com li chastelains de Couci,

Qui au cuer n'avoit s'amor non ${ }^{19}$.

Pour qualifier les sentiments du héros chanteur, l'auteur lui a donc permis de se comparer à un trouvère connu pour ses qualités d'amant mais aussi de musicien : le chevalier du roman endosse ainsi, par son affinité de sentiments avec le châtelaintrouvère, une part de sa personnalité et par là-même de sa réalité historique.

La chanson citée à la fin du Tractatus de matrimonio scientiarum, tranposition en quatrain d'alexandrins monorimes du Mariage des sept arts octosyllabique de Jehan le Teinturier d'Arras est attribuée dans le seul chansonnier $C$ qui la conserve au même trouvère, ce qui confirme la référence historico-littéraire initiale du poème, postérieure de peu seulement à son modèle

Les citations d'Adam de la Halle, dans la Panthere de Nicole de Margival (écrit entre 1290 et 1328 environ) et la traduction du Ludus super Anticlaudianum ${ }^{20}$

${ }^{19}$ La Châtelaine de Vergy, éd. par Jean Dufournet et Liliane Dulac, Paris, Gallimard, 1994. Vers 291-293. 
(effectuée à la toute fin du XIII ${ }^{\mathrm{e}}$ siècle ou dans les premières années du $\mathrm{XIV}^{\mathrm{e}}$ ) constituent pour ces textes une référence relativement récente, le trouvère arrageois ayant été actif entre le milieu du XIII ${ }^{\mathrm{e}}$ siècle et 1288 (1306 pour certains, cette hypothèse étant toutefois difficile à vérifier ${ }^{21}$ ). Pour Nicole, la citation historique d'un trouvère renommé se double vraisemblablement d'une volonté de reconnaissance de sa propre œuvre poétique: les chansons d'Adam de la Halle côtoient en effet les siennes propres, comme pour donner à ses créations lyriques un poids supplémentaire vis à vis de ses lecteurs. Le rôle de «témoin d'un temps musical » paraît ainsi donc quelque peu intéressé à sa future renommée...

Quant à celles proposées par le trouvère lui-même dans ses jeux théâtraux, elles introduisent une distance importante par rapport au reste de la musique insérée par Adam, qu'elle soit son fait ou celui de la mode du temps : la chanson de geste Audigier, dont un fragment est noté dans Robin et Marion date de la fin du XII siècle ou du début du XIII ${ }^{\mathrm{e}}$; le vers de chanson de toile (anonyme) conservé dans la Feuillee renvoie également à un temps ancien, au mieux celui de Guillaume de Dole dans lequel les mêmes chansons constituaient, de l'aveu de leurs chanteuses, des manières d'anachronismes musicaux. Ces citations d'une histoire musicale déjà ancienne sont en outre - et ce n'est peut-être pas un hasard - nettement teintées de satire. Audigier est en soi parodique et scatologique et la présentation dans la Feuillee du fragment de toile est elle-même caricaturale, l'aubergiste enjoignant Adam et Hane de «recaner» (littéralement : «braire») la chanson, afin de témoigner de leur art.

L'étude des origines poétiques des chansons connues citées dans certaines œuvres narratives et didactiques du XIII ${ }^{\mathrm{e}}$ siècle et du début du $\mathrm{XIV}^{\mathrm{e}}$ permet donc de constater, la plupart du temps, une antériorité marquée des références musicales par rapport à leur mention ultérieure. Tout se passe comme si les auteurs narratifs avaient choisi des poètes dont la renommée était déjà établie, s'abritant derrière elle pour justifier en quelque sorte leur récit, ou tout au moins l'étayer. La seule exception notable à cet état général, hormis la mention unique par Jean Renart d'un troubadour contemporain, se produit avec Gerbert de Montreuil et son roman de la Violette. Il est le seul à insérer des chansons de trouvères choisis parmi ses contemporains et à rendre ainsi garants de la vérité de son récit des poètes dont la renommée n'est pas encore à son époque aussi bien établie que celle de certains autres, plus anciens, qui les côtoient. La mémoire musicale des romanciers, celle qu'ils désirent laisser à leurs lecteurs, est donc le plus souvent une mémoire déjà ancienne, ce qui rejoint la préoccupation souvent avérée par les auteurs de justifier leur conte par une version antérieure, gage de sa véracité. Cette précaution prise, en

\footnotetext{
${ }^{20}$ Dans ce texte, je n'ai pu retrouver que cette seule référence à un poète connu. C'est la chanson R 1661a Puis que je suy de l'amoureuse loy, contrafactum de R 1661, portant le même titre, d'Adam.

${ }^{21}$ Le nom de «Maistre Adam le Boscu» apparaît en effet à cette date dans le rôle de l'Échiquier, parmi les nombreux jongleurs et ménestrels venus assister, à Westminster, à l'adoubement d'Édouard d'Angleterre. On lira à ce sujet l'article de Fabienne Gégou, «Adam le Bossu était-il mort en 1288 ?», Romania, 86, 1965, p. 111-117.
} 
revanche, certains romanciers ${ }^{22}$ n'hésitent toutefois pas à mêler leurs propres compositions à celles de leurs trouvères de prédilection, replaçant ainsi dans un temps réellement contemporain l'histoire et la ramenbrance musicales de leurs œuvres.

Anne Ibos-Augé

${ }^{22}$ Jean Renart, probablement, avec la laisse de Gerbert de Metz, Jakemes avec l'ultime chanson du roman, Sans faindre voel obeir, Girart d'Amiens avec la chanson du duc de Galice, Nicole de Margival avec ses propres ballades et rondeaux en sont quelques exemples. 